\title{
Contribuição do solo natural na drenagem urbana em uma área suscetível a
}

\section{alagamentos no Recife - PE}

\author{
Contribution of natural soil to urban drainage in an area susceptible to flooding in Recife - PE \\ Contribución del suelo natural al drenaje urbano en una zona susceptible a inundaciones en Recife
}

- PE

Recebido: 16/05/2021 | Revisado: 29/05/2021 | Aceito: 29/05/2021 | Publicado: 13/06/2021

Diogo Botelho Correa de Oliveira

ORCID: https://orcid.org/0000-0003-1556-5664

Universidade Federal de Pernambuco, Brasil E-mail: diogo.botelho@ufpe.br

Willames de Albuquerque Soares ORCID: https://orcid.org/0000-0003-3268-7241 Universidade de Pernambuco, Brasil E-mail: was@poli.br

Simone Rosa da Silva

ORCID: https://orcid.org/0000-0001-7138-7546 Universidade de Pernambuco, Brasil E-mail: simone@poli.br

\begin{abstract}
Resumo
Os eventos pluviométricos extremos são causadores de diversas tragédias e transtornos. A drenagem urbana é de essencial relevância para combater estes problemas. Nem sempre as galerias urbanas conseguem absorver toda a demanda oriunda destes eventos, logo o maior aliado dessas é o solo natural. Poucos estudos visam quantificar a parcela de contribuição desse meio na drenagem nos centros urbanos. Este estudo teve como finalidade estimar o dimensionamento necessário das galerias pluviais para sanar estes problemas, utilizando os parâmetros do solo de taxa de infiltração e a capacidade volumétrica de retenção das águas pluviais, analisando o balanço hídrico em uma área comercial e academicamente importante da cidade do Recife - PE. Foram utilizados ensaios de granulometria e de infiltração a anel simples para modelar o balanço da água nas regiões de terreno natural da área pré-determinada. O solo mostrou-se bom condutor, sendo capaz de absorver chuvas diárias de quase $49 \mathrm{~mm}$ em seu próprio limite físico. A dificuldade encontrada foram os baixos índices de terreno natural disponíveis na região - 17\% do total, reduzindo sua atuação. Verificou-se que seriam necessários aproximadamente 410 1/s como vazão necessária da rede principal dessa área urbana, para absorver a maior parte dos eventos extremos de precipitação.
\end{abstract}

Palavras-chave: Escoamento superficial; Infiltração de água no solo; BEST; Eventos pluviométricos extremos.

\begin{abstract}
Extreme rainfall events are the cause of several tragedies and disorders. Urban drainage is essential to combat these problems. Urban galleries are not always able to absorb all the demand arising from these events, so the greatest ally of these is natural soil. Few studies aim to quantify the contribution of this medium to drainage in urban centers. This study aimed to estimate the necessary dimensioning of storm sewers to solve these problems, using the soil parameters of infiltration rate and the volumetric capacity of rainwater retention, analyzing the water balance in a commercial and academically important area of the city of Recife PE. Granulometry and simple ring infiltration tests were used to model the water balance in the regions of natural terrain in the predetermined area. The soil proved to be a good conductor, being able to absorb daily rainfall of almost $49 \mathrm{~mm}$ in its own physical limit. The difficulty encountered was the low rates of natural terrain available in the region - $17 \%$ of the total, reducing its performance. It was found that approximately $410 \mathrm{l} / \mathrm{s}$ would be needed as the necessary flow from the main network of this urban area, to absorb most of the extreme precipitation events.
\end{abstract}

Keywords: Surface runoff; Infiltration of water in the soil; BEST; Extreme rainfall events.

\section{Resumen}

Los eventos de lluvias extremas son la causa de varias tragedias y desórdenes. El drenaje urbano es fundamental para abordar estos problemas. Las galerías urbanas ni siempre son capaces de absorber toda la demanda derivada de estos eventos, por lo que su mayor aliado es el suelo natural. Pocos estudios pretenden cuantificar la contribución de este medio al drenaje en los centros urbanos. Este estudio tuvo como objetivo estimar el dimensionamiento necesario de las galerías de lluvia para remediar estos problemas, utilizando los parámetros del suelo de tasa de infiltración y la capacidad 
volumétrica de retención de agua de lluvia, analizando el balance hídrico en un área comercial y académicamente importante de la ciudad de Recife PE. Se utilizaron pruebas de granulometría e infiltración de anillos simples para modelar el balance hídrico en regiones de terreno natural en el área predeterminada. El suelo demostró ser un buen conductor, pudiendo absorber lluvias diarias de casi $49 \mathrm{~mm}$ en su propio límite físico. La dificultad encontrada fueron las bajas tasas de terreno natural disponible en la región - 17\% del total, reduciendo su rendimiento. Se encontró que se requerirían aproximadamente $410 \mathrm{l} / \mathrm{s}$ como caudal necesario de la red principal de esta área urbana, para absorber la mayoría de los eventos de precipitación extrema.

Palabras clave: Escorrentía superficial; Infiltración de agua en el suelo; BEST; Eventos de lluvia extrema.

\section{Introdução}

Observa-se na cidade do Recife, Pernambuco (PE), ao longo dos anos, que nos períodos de chuvas são recorrentes as cheias e alagamentos de origem pluvial. As galerias de drenagem urbana são as principais agentes de escoamento de água, mas devido ao planejamento pouco adequado para a atualidade e à falta de manutenção adequada, elas não são suficientes e acabam sendo subaproveitadas e incapazes de suprir a demanda exigida em eventos pluviométricos aos quais foram dimensionadas. O solo natural é o maior aliado das galerias neste âmbito, já que a água da chuva infiltra e percola este meio, sendo direcionada para suas camadas mais internas, reduzindo o saldo que escoa superficialmente no ambiente urbano (Holanda et al., 2020; Melo et al.,2013).

Devido à porosidade do solo, parte da água que entra em contato com sua superfície é infiltrada, atingindo picos máximos de fluxos quando sua condutividade hidráulica atinge a saturação e decresce gradativamente de acordo com a saturação superficial do solo, convergindo para sua taxa residual. É de alta relevância conhecer determinadas propriedades hidráulicas do solo - como a condutividade hidráulica e a capacidade de infiltração - para quantificar o volume de água retido por ele (Soares et al., 2005; Souza et al., 2008; Almeida et al.,2012).

Segundo estudo de Costa et al. (2016), os alagamentos em São Luís - MA estão associados às áreas de baixa declividade e de alto crescimento urbano. Fagundes (2002) avaliou qualitativamente a relação entre o crescimento urbano e demográfico com a impermeabilização do solo, em Porto Alegre - RS, concluindo que a descaracterização do solo é um fator de alta influência para contribuição de enchentes e desastres associados ao escoamento superficial da água.

Alguns trabalhos realizados no Recife/PE buscam analisar o impacto da impermeabilização do solo causada pelo avanço urbano. Moura e Silva (2015) mostram que, nos últimos 40 anos, está descaracterização do solo natural cresceu de 37,17\% para 73,13\%. Analisaram também alternativas que buscam minimizar o impacto causado, como a utilização de jardins de chuva e pavimentos permeáveis. Oliveira et al (2015) também observa a crescente redução de áreas vegetadas em grande parte da cidade, reforçando a importância da manutenção dessas regiões e a sua melhor distribuição para otimizar o aproveitamento e a atuação destas na amenização dos impactos sofridos. Devido aos constantes problemas causados pelas chuvas fortes nesta região, a necessidade de desenvolver alternativas que intensifiquem a atuação do solo na drenagem urbana torna-se de extrema importância, reafirmando sua relevância para tal finalidade (Holanda, Soares e Oliveira, 2020).

O ensaio de infiltração de água no solo utilizando anel simples aplicado com intuito de obter parâmetros hidrodinâmicos do solo, como a condutividade hidráulica saturada, a taxa de infiltração de água e demais indicadores edáficos (Oliveira, 2018). Souza et al, 2013, com objetivo de estimar o balanço hídrico que ocorre no solo, utilizou-se do método em latossolo amarelo. Para caracterizar hidrodinamicamente o solo, Netto et al, 2013, combinou dados coletados em campo com uma modelagem numérica a partir da mesma metodologia, classificando-a como eficiente e fielmente representativa para a finalidade desejada. Silva Júnior et al (2016) destaca a simplicidade e eficiência do ensaio de infiltrômetro de anel simples para determinar a infiltração em modelos chuva-vazão, com o objetivo de obter os principais parâmetros que influenciam diretamente no escoamento superficial de uma região.

As curvas IDF (Intensidade, Duração e Frequência) são largamente utilizadas para caracterização de precipitações 
intensas, que são as que frequentemente causam mais danos materiais e humanos em desastres associados a eventos pluviométricos, para previsão e dimensionamento de redes de drenagem urbana (Bielenki Júnior et al., 2016). Através destas é possível caracterizar hidrologicamente a bacia desejada, estimando os hidrogramas, hietogramas e demais informações relevantes de estudo.

Apesar dos avanços neste tema, poucos estudos na cidade do Recife visam quantificar o potencial hídrico do solo de retenção de água, obtendo dados mais precisos para auxiliar na gestão e planejamento de dimensionamentos de galerias de drenagem e técnicas compensatórias sustentáveis que visem minimizar ou sanar os transtornos causados em períodos chuvosos. Este trabalho tem por objetivo estimar o fluxo necessário para reduzir o escoamento superficial em eventos pluviométricos extremos, considerando a contribuição do solo natural na drenagem urbana, através de comparações entre as curvas de infiltração de água no solo, obtidas através de ensaios de infiltração utilizando o infiltrômetro de anel simples, e de volume acumulado de precipitação em uma região relevante da cidade, além de avaliar o método BEST utilizado em terrenos característicos da cidade do Recife.

\section{Metodologia}

O bairro da Madalena - Recife/PE é um dos mais importantes da cidade, pois abriga vários pontos importantes da urbe, como o estádio Adelmar da Costa Carvalho, o Museu da Abolição, os campi Benfica da Universidade de Pernambuco, centros de compras, entre outros. Também serve de integração do centro da cidade com a Universidade Federal de Pernambuco (UFPE) e o Terminal Rodoviário do Estado (TIP-PE). Na Figura 1 pode ser visualizada a distribuição espacial do bairro, situada na região mais central da capital pernambucana.

Figura 1. Distribuição espacial do bairro da Madalena, local deste estudo.

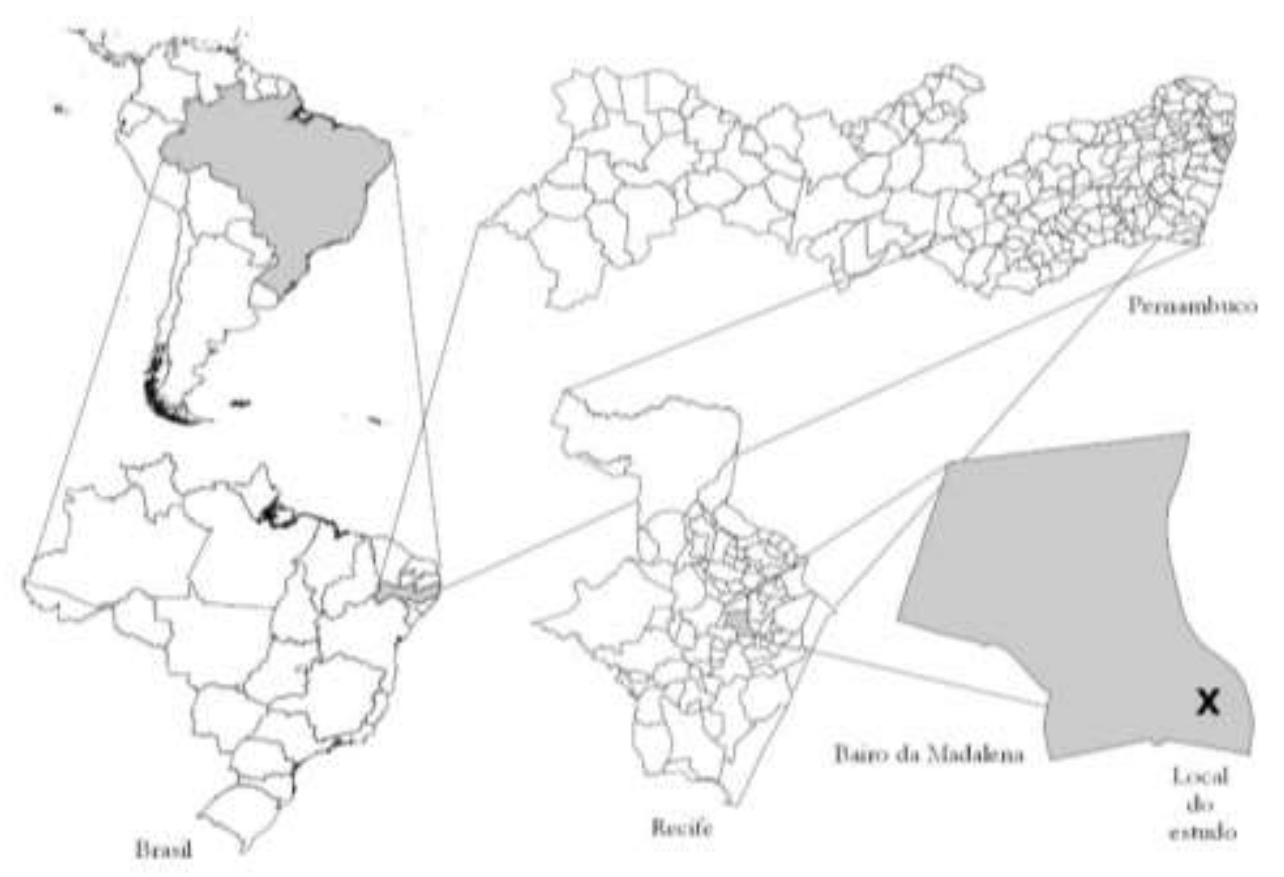

Fonte: Autores. 
A bacia de estudo delimitada é apresentada na Figura 2, sendo alvo constante de alagamento nos períodos de chuva, trata-se de uma região de grande interesse social. Compreendendo cerca de $228.000 \mathrm{~m}^{2}$, a área localiza-se nos arredores da Escola Politécnica de Pernambuco, onde aproximadamente 17\% desta é composta por solo natural. As regiões de 1 a 9 indicam as principais ocorrências deste. Foram realizados ensaios de infiltração de água no solo in loco, granulometria do solo, determinação da densidade aparente e das umidades naturais e saturadas do solo.

Figura 2. Região de estudo delimitada, indicando pontos de solo natural, onde foram realizados os ensaios e coletadas amostras.

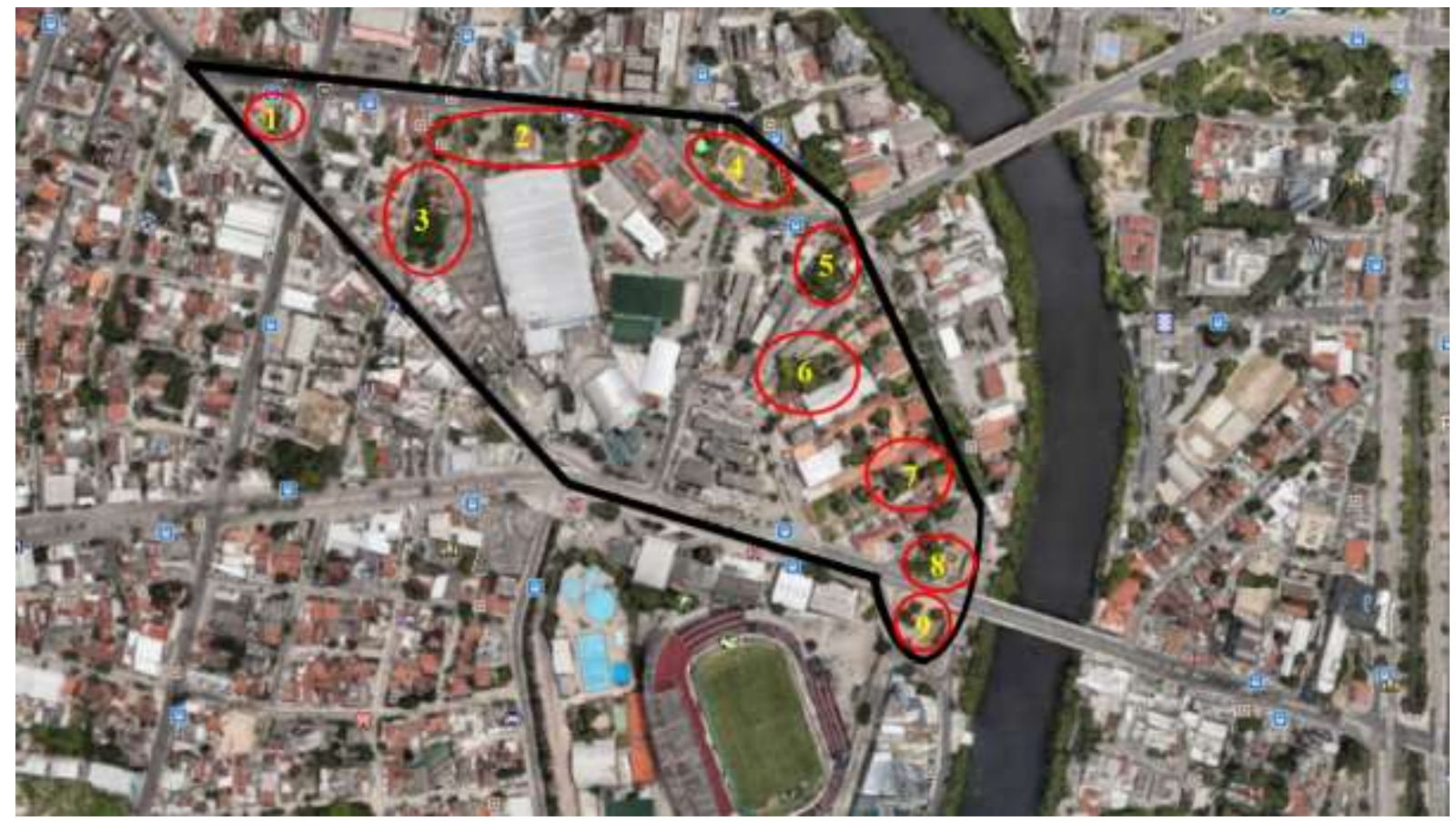

Fonte: Autores.

\subsection{Parâmetros do local}

Os acumulados mensais de precipitação, disponibilizados pela Agência Pernambucana de Águas e Clima (APAC), durante os anos de 1966 e 2016 estão representados na Figura 3. Os dados foram indicados de acordo com a estação do Curado, já que esta é a mais próxima (cerca de $2 \mathrm{~km}$ ) do bairro da Madalena que disponibilizava a maior e mais antiga quantidade de registros. Os maiores índices pluviométricos são observados entre os meses de abril e julho, indicando a maior frequência de incidentes nestes meses.

Com base em dados semelhantes, da mesma região de estudo, Silva e Araújo (2013), determinaram a equação IDF para a cidade do Recife, analisando a mesma em diversos tempos de retornos utilizados para dimensionamento de macro e micro drenagem urbana. Como resultado, a equação encontrada é descrita a seguir:

$$
I_{n}=\frac{1380,217 x T R^{0,19869}}{(t+22)^{0,7821}}
$$

Onde $T R$ é o tempo de retorno em anos, $t$ é o tempo de duração da chuva, em minutos, na região onde abrange o estudo e $I_{n}$ é a intensidade da chuva correspondente ao tempo de duração da mesma em $\mathrm{mm} / \mathrm{h}$. 
Figura 3. Média acumulada mensal do histórico de chuvas, em milímetros, durante os meses do ano.

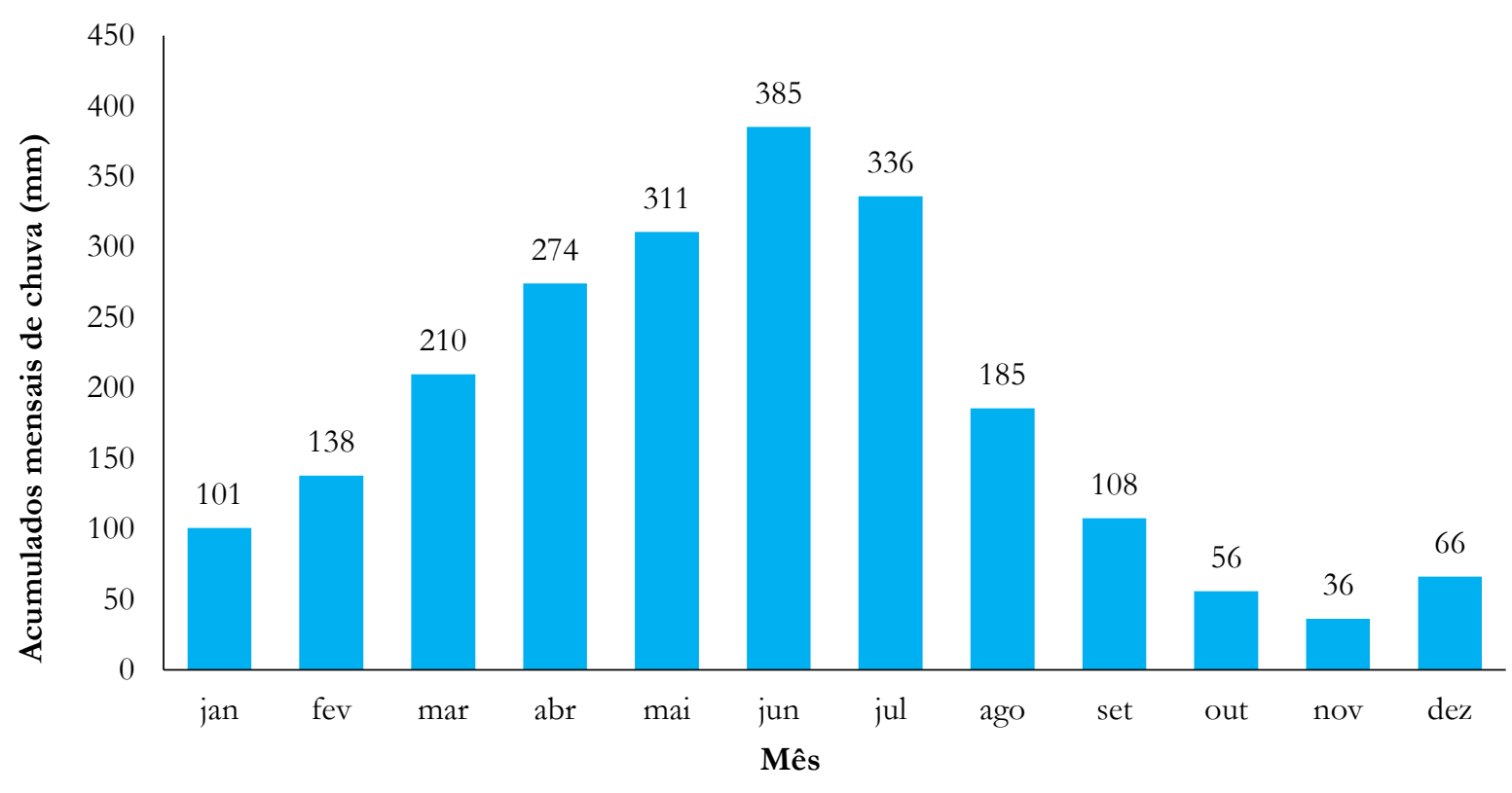

Fonte: Autores.

A partir dos mesmos dados, fornecidos por Silva e Araújo (2013), foi possível construir as hipérboles IDF de recife, conforme mostra a Figura 4.

Figura 4. Curvas IDF de Recife para os tempos de retorno de 2, 3, 5 e 10 anos, que são as mais utilizadas para o dimensionamento de microdrenagens.

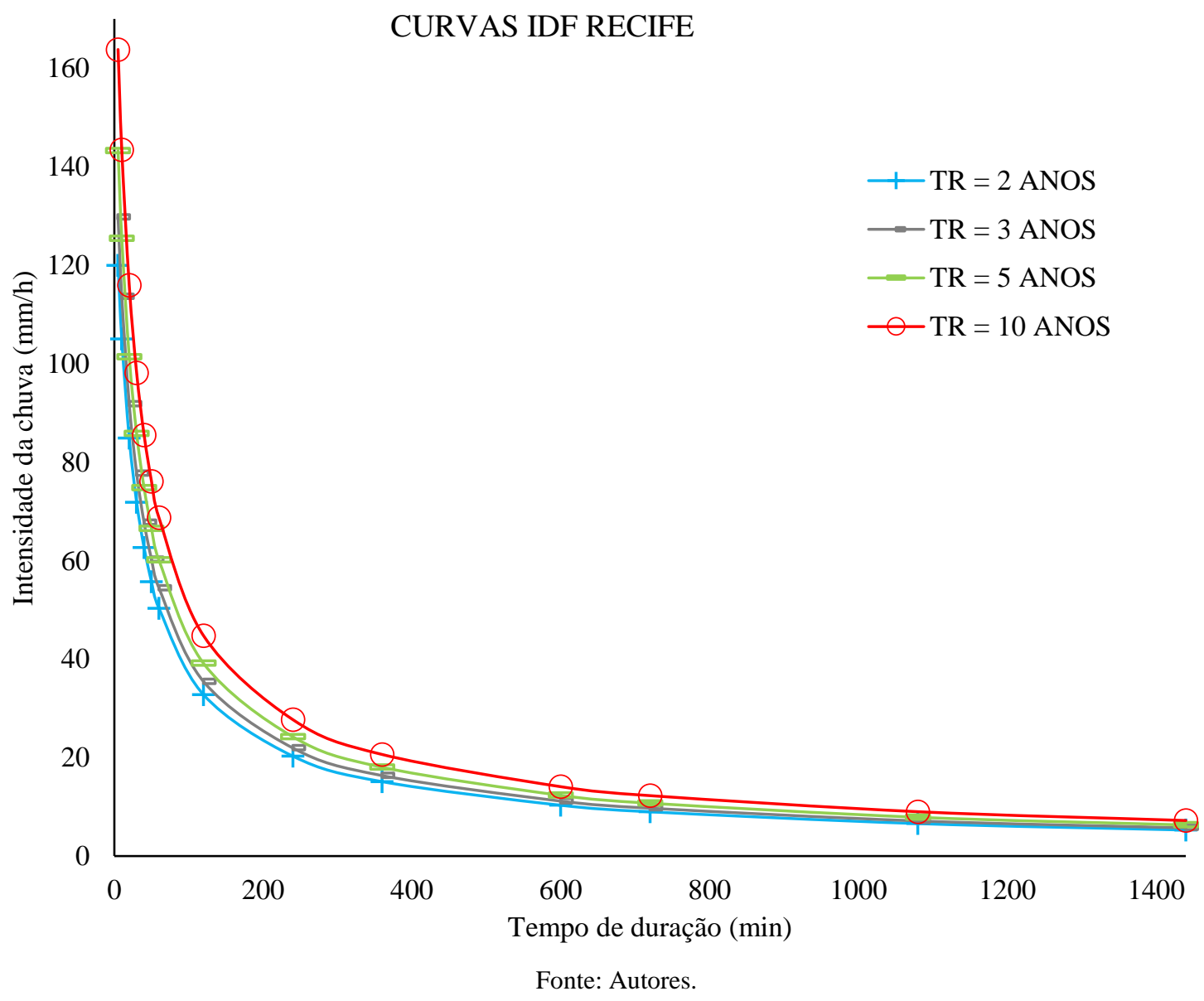


Com a bacia hidrográfica delimitada, é possível aplicar a metodologia descrita acima. Para este estudo, é considerada como valor de entrada a água de origem pluvial, e de saída a decorrente da infiltração da água do solo, a fim de analisar a influência desta na bacia delimitada. Para isto, foi necessário obter a precipitação acumulada de chuva oriunda do hietograma de projeto para um tempo de retorno de 5 anos e de 10 anos. A curva de infiltração de água acumulada no solo foi encontrada com utilização do método BEST (Lassabatére et al., 2006) descrito em tópico posterior, sendo definida a expressão matemática correspondente à infiltração pela Equação 5.

\subsection{Dados Pluviométricos}

A partir de metodologia indicada por Bertoni e Tucci (2007), com os dados disponíveis através das curvas IDF, é possível obter os hietogramas e hidrogramas de projeto, esta última através do método racional, definida pela Equação 2, por se tratar de uma bacia inferior a $2 \mathrm{~km}^{2}$ (Porto, 1995), simulando chuvas intensas para o tempo de retorno adotado, que neste caso é de 5 anos e de 10 anos, por se tratar de uma área comercialmente importante e que abriga diversos lugares de interesse público, para assim obter a curva de volume acumulado de água, para fins comparativos com a curva de infiltração acumulada de água no solo.

$$
Q=0,278 C I_{n} H
$$

Para $i$ definido como a intensidade de precipitação, em mm/h, e $H$ a área da bacia delimitada, em $\mathrm{km}^{2}$. O $C$ é o coeficiente de escoamento superficial, sendo adimensional e variando de 0 a 1 . Como o interesse é maximizar o volume de chuva precipitado e aumentar a segurança de projeto, foi adotado o tempo de duração de chuva de 60 min. (Bidone \& Tucci, 1995; Souza, 2014).

\subsection{Ensaio de Infiltração}

Como mostrado na Figura 5, o ensaio consistiu no infiltrômetro de anel simples, cravado a $1 \mathrm{~cm}$ de profundidade da superfície, visando minimizar a interferência de fatores estruturais e externos, garantindo fluxo vertical na superfície do solo. Os tempos foram cronometrados para que ocorra a infiltração total de volumes constantes de água no interior do anel até atingir a saturação do solo, onde o fluxo se torna praticamente constante, determinando-se a vazão da água no solo. 
Figura 5. Ensaio de Infiltrômetro de anel simples, realizado dentro da área de estudo.

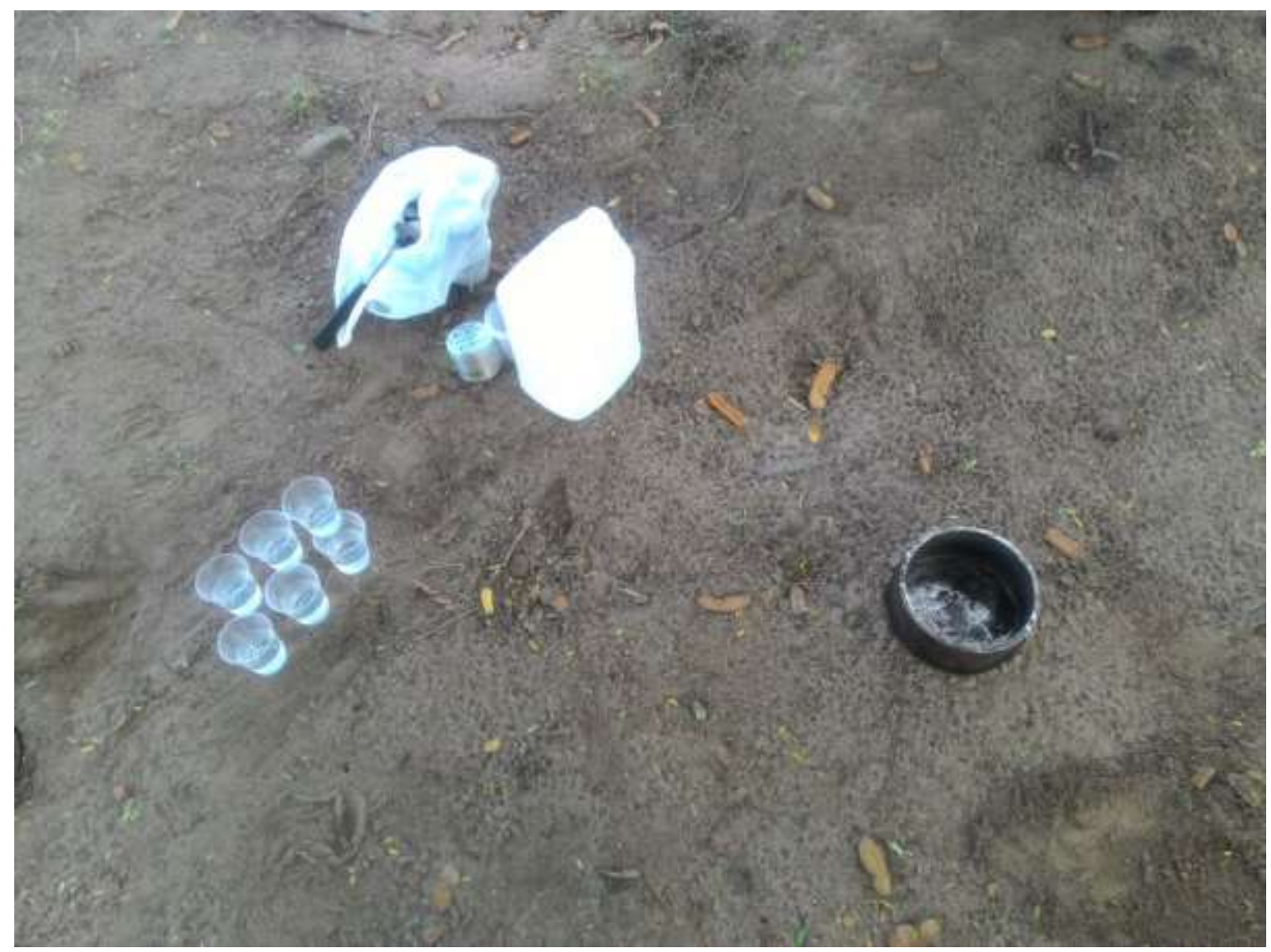

Fonte: Autores.

Para estimar a condutividade hidráulica $(K)$, condutividade hidráulica saturada $\left(K_{S}\right)$ e a Sorvidade $(S)$, utilizou-se a Equação proposta por Harverkamp et al., em 1994:

$$
I_{3 D}=\left(K+\frac{\gamma S^{2}}{r_{d\left(\theta_{f}-\theta_{i}\right)}}\right) t+\frac{S^{2}}{2 K_{S}(1-\varepsilon)} \ln \left(\frac{1}{\varepsilon}\right)
$$

Em que $\gamma$ é um parâmetro que traduz os efeitos da gravidade na geometria tridimensional da infiltração, dentro do intervalo de 0,6 a 0,8; para Smettem et al. (1994) o valor de 0,75 é o mais adequado; $\varepsilon$ uma constante dentro do intervalo $0<\varepsilon<1$; $r_{d}$ é o raio do disco, $\theta_{f}$ e $\theta_{i}$, são as umidades volumétricas final e inicial, respectivamente.

A determinação destes três parâmetros citados anteriormente é de grande importância para a estimativa do volume total de água infiltrado em diferentes períodos de tempo. Oliveira e Soares (2017) realizaram a análise de modelos de infiltração de água no solo para esta mesma região, concluindo que o modelo BEST (Beerkan Estimation of Soil Parameters) é o mais indicado para esta finalidade, neste local de estudo.

O modelo BEST (Lassabatère et al., 2006) é o mais moderno e mais complexo modelo empírico de infiltração até o momento, já que ele utiliza o ensaio a anel simples e o resultado da granulometria do solo estudado para estimar os parâmetros necessários para seu desenvolvimento. Este modelo utilizou-se especificamente do proposto por Van Genuchten, com o objetivo de determinar os parâmetros $\theta_{\mathrm{r}}, \theta_{\mathrm{s}}, \mathrm{n}, \alpha$, com a Equação 3 (Van Genuchten, 1980): 


$$
\begin{gathered}
\theta=\theta_{n}+\frac{\theta_{0}-\theta_{n}}{\left[1+|a \psi|^{n}\right]^{1-\frac{1}{n}}} \\
m=1-\frac{2}{n}
\end{gathered}
$$

Para $n, m$ e a como parâmetros de forma ajustados, $\theta_{0}$ é a umidade saturada, $\theta_{n}$ é a umidade residual e $\Psi$ é um parâmetro de ajuste.

De Haverkamp et al. (1994), foram utilizadas as equações de infiltração tridimensional acumulada e da taxa de infiltração, descritas por:

$$
\begin{aligned}
& I=S t^{\frac{1}{2}}+\left(A S^{2}+B K_{s}\right) t \\
& q=\frac{S}{2 \sqrt{t}}+\left(A S^{2}+B K_{s}\right)
\end{aligned}
$$

Considerando grandes variações de tempo, temos:

$$
\begin{gathered}
I_{+\infty}(t)=\left(A S^{2}+K_{s}\right) t+C \frac{S^{2}}{K_{S}} \\
q_{+\infty}=A S^{2}+K_{s}
\end{gathered}
$$

Onde as constantes A, B e C são dependentes das condições iniciais:

$$
\begin{gathered}
A=\frac{\gamma}{r_{d}\left(\theta_{0}-\theta_{n}\right)} \\
B=\frac{1,4}{3}\left[1-\left(\frac{\theta_{n}}{\theta_{0}}\right)^{\eta}\right]+\left(\frac{\theta_{n}}{\theta_{0}}\right)^{\eta} \\
C=\frac{1}{0,8\left[1-\left(\frac{\theta_{n}}{\theta_{0}}\right)^{\eta}\right]} \ln \left(\frac{1}{0,6}\right)
\end{gathered}
$$

Em que $\gamma$ é 0,75 para a maioria dos solos onde $\theta_{n}<0,25 \theta_{0}$ (Smetten et al., 1994; Haverkamp et al., 1994), $\eta$, é um parâmetro de forma, $r_{d}$ é o raio do anel utilizado.

A sorvidade pode ser estimada a partir da condutividade hidráulica saturada, considerando as umidades inicial e final pela equação 12 :

$$
S^{2}\left(\theta_{n}, \theta_{0}\right)=-c_{p} \theta_{0} K_{s} h_{g}\left(1-\frac{\theta_{n}}{\theta_{0}}\right)\left[1-\left(\frac{\theta_{n}}{\theta_{0}}\right)^{\eta}\right]
$$

Para $c_{p}$ e $h_{g}$ como parâmetros de escala, define-se por: 


$$
\begin{gathered}
c_{p}=\Gamma\left(1+\frac{1}{n}\right)\left[\frac{\Gamma\left(m \eta-\frac{1}{n}\right)}{\Gamma(m \eta)}+\frac{\Gamma\left(m \eta+m-\frac{1}{n}\right)}{\Gamma(m \eta+m)}\right] \\
h_{g}=-\frac{s^{2}}{c_{p}\left(\theta_{0}-\theta_{n}\right)\left[1-\left(\frac{\theta_{n}}{\theta_{0}}\right)^{\eta}\right] K_{S}}
\end{gathered}
$$

Onde $\Gamma$ é a função gama.

Portanto, para estimar a curva de infiltração de água no solo pelo método proposto, além do ensaio de infiltração a anel simples, é necessário utilizar-se dos resultados dos ensaios de granulometria, densidade aparente, umidade residual e umidade saturada.

\subsection{Granulometria e Caracterização hidrodinâmica}

Para determinar a granulometria do solo utilizou-se o método do densímetro, para assim obter os valores numéricos dos parâmetros necessários. O referido método foi realizado de acordo com o manual de métodos de análise do solo, elaborado pela EMBRAPA (Teixeira et al., 2017). As umidades residuais e saturadas foram realizadas através do método da estufa, também referenciada pelo mesmo manual.

Já a densidade aparente foi obtida através do trado de Uhland, já que possui baixos índices de erro para obtenção deste índice (Souza et al., 2014).

\subsection{Capacidade de retenção acumulada de água do solo e voluma acumulado de chuva}

Para calcular o volume total retido pelo solo é necessária a equivalência entre a amostra representativa da infiltração da água no solo e sua área correspondente. Este produto mostra a vazão associada àquela região. A relação entre volume e vazão é dependente do tempo, logo, considera-se o tempo de infiltração no cálculo de quantificação total de água disposta no local demarcado. Sendo $I$ a taxa de infiltração $(\mathrm{mm} / \mathrm{s}), H\left(\mathrm{~m}^{2}\right)$ a área correspondente a esta variável e $t$ o tempo (s), a capacidade volumétrica $V(I)$ de retenção do solo, em $\mathrm{m}^{3}$, é expressa por:

$$
V(I)=\int I d t * H \cdot 10^{-3}
$$

Semelhante ao desenvolvimento utilizado anteriormente, é possível descrever o volume acumulado de águas pluviais, a partir do hietograma, da seguinte maneira:

$$
V(A p)=\int \operatorname{Pr} d H
$$

Onde $I$ é expresso em $\mathrm{mm}$, a área discretizada $\mathrm{em}^{2}$ e $V(A p) \mathrm{em}^{3}$.

\section{Resultados e Discussão}

Os resultados do ensaio de granulometria estão apresentados na Figura 6, identificando-se os percentuais de areia, silte e argila e os valores das condutividades hidráulicas saturadas de cada uma das provas coletadas. Segundo classificação de solos da EMBRAPA (2018), as amostras coletadas nos pontos 3, 5 e 9 foram classificadas como arenosas, a amostra do ponto 4 foi classificada como areia franca, e as demais foram classificadas como franco arenosas. Estas classificações indicam a capacidade do terreno de possuir alta condutividade e alto potencial de retenção de chuvas. 
As amostras se mostraram com composições semelhantes entre si, destacando a amostra 5, que possuiu condutividade mais elevada que as demais. Isto corrobora com a granulometria, pois este solo possui a maior fração de areia e a menor fração de argila identificada em relação às demais.

Figura 6. Percentuais de areia $(A)$, silte $(S)$ e argila $(G)$ e condutividades hidráulicas saturadas de cada amostra analisada.

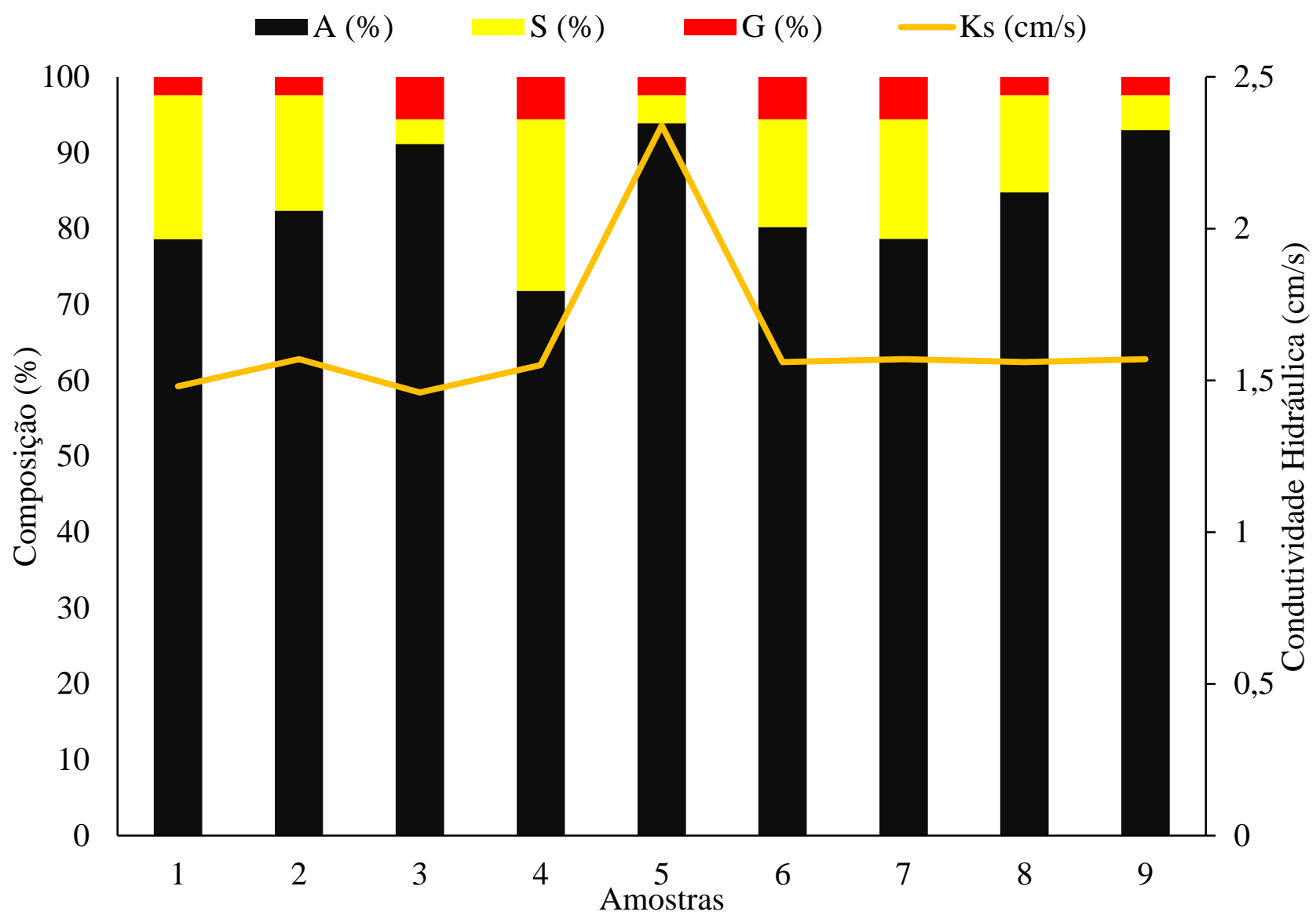

Fonte: Autores.

A partir dos resultados obtidos dos ensaios de infiltração com utilização do anel simples e de granulometria, é possível construir a curva de infiltração acumulada de água no solo para cada local estudado, conforme indicado na Figura 7. Como os ensaios apresentaram resultados semelhantes, foi desenvolvida uma curva de infiltração média, ponderando-se as influências de cada região natural de acordo com sua área, como mostra a Equação 17:

$$
I_{m}=\frac{\sum_{i=1}^{9} I_{n} H_{n}}{\sum_{i=1}^{9} H_{n}}
$$

Onde $I_{m}$ é a infiltração média acumulada do solo, em mm; $I_{n}$ é a infiltração correspondente de cada localidade para o tempo correspondente, em $\mathrm{mm} ; H_{n}$ é a área equivalente de cada localidade com solo natural, em $\mathrm{m}^{2}$.

Cecílio (2005) avaliou 3 tipos de solos em diferentes localidades na região de Viçosa - MG. Em testes de laboratório, identificou-se que os acúmulos de volumes infiltrados dos solos estudados são da ordem de $130 \mathrm{~mm}$ para 30 min de infiltração em áreas de caráter rural, indicando alto potencial de infiltração. 
Figura 7. Curva média de infiltração acumulada de água no solo para o local de estudo.

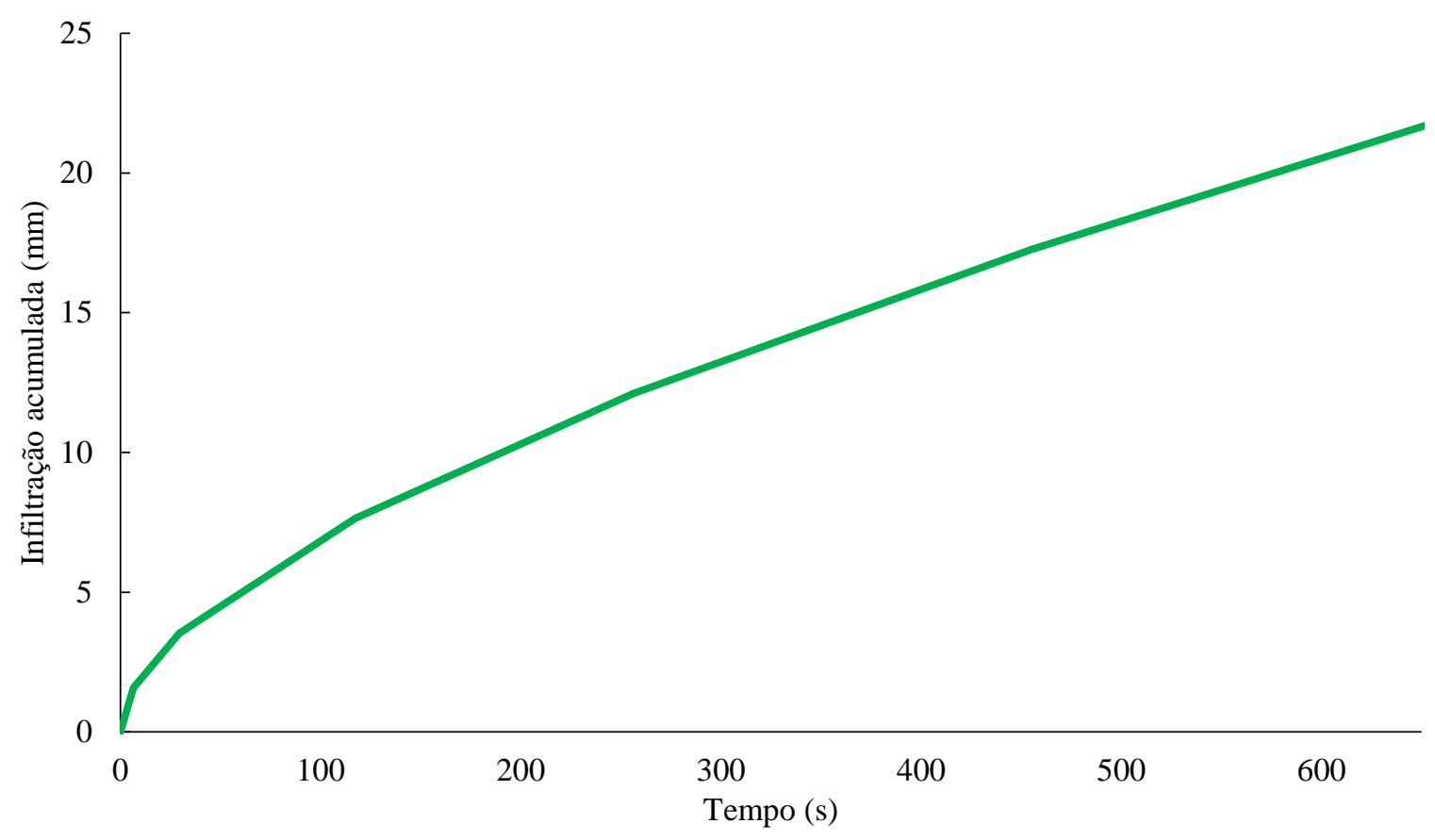

Fonte: Autores.

A partir das equações IDF estabelecidas para a cidade do Recife, resultaram nos hietogramas das Figuras 8 e 9, para os tempos de retorno de 5 anos e de 10 anos, respectivamente, adequados às condições previamente estabelecidas da localidade do estudo. A partir destes dados, foi possível estabelecer a curva de volume acumulado de precipitação, em função da área total delimitada $\left(228.000 \mathrm{~m}^{2}\right)$, conforme mostra a Figura 10.

Os maiores acúmulos de águas pluviais são identificados logo após os picos de precipitação dos hietogramas. Na medida em que o tempo decorrido se aproxima do tempo de concentração, as diferenças entre os volumes de tempo de retorno de 5 e 10 anos vão se tornando mais consideráveis, e neste caso em específico atingindo cerca de $2000 \mathrm{~m}^{3}$. A contribuição do solo natural se apresenta sensível, atingindo volumes máximos de retenção, para o tempo de concentração, de cerca de $4000 \mathrm{~m}^{3}$. Um fator que limita a maior contribuição do mesmo é a área disponível para infiltração, que é apenas de 17\% da área total, sendo que o aconselhável pela LUOS (Lei de Uso e Ocupação do Solo), 1996, do Recife determina como mínimo para o bairro da área estudada em $25 \%$ de determinada área total. 
Figura 8. Hietograma de projeto para o tempo de retorno de 5 anos, considerando-se a IDF utilizada.

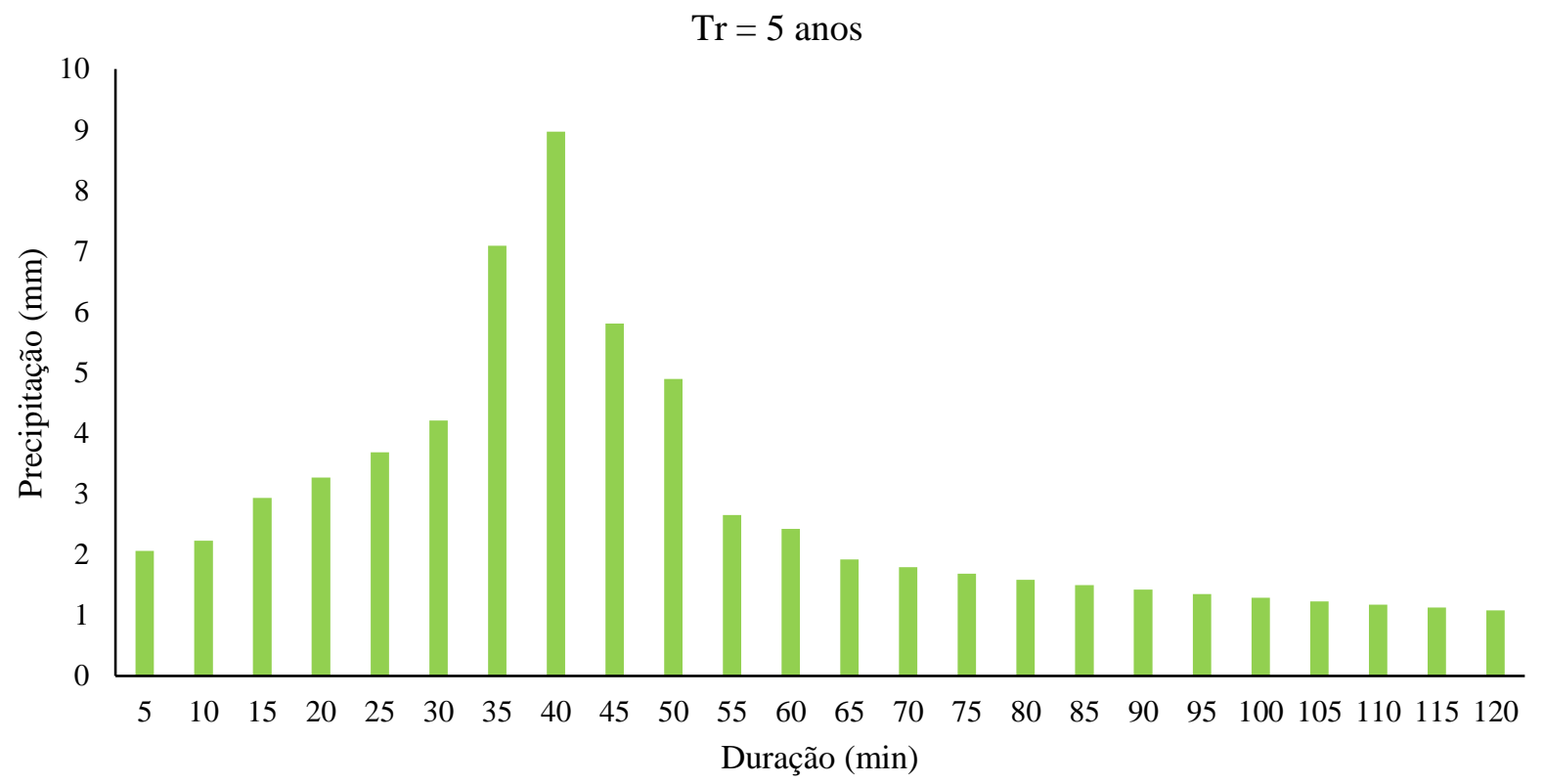

Fonte: Autores.

Figura 9. Hietograma de projeto para o tempo de retorno de 10 anos, considerando-se a IDF utilizada.

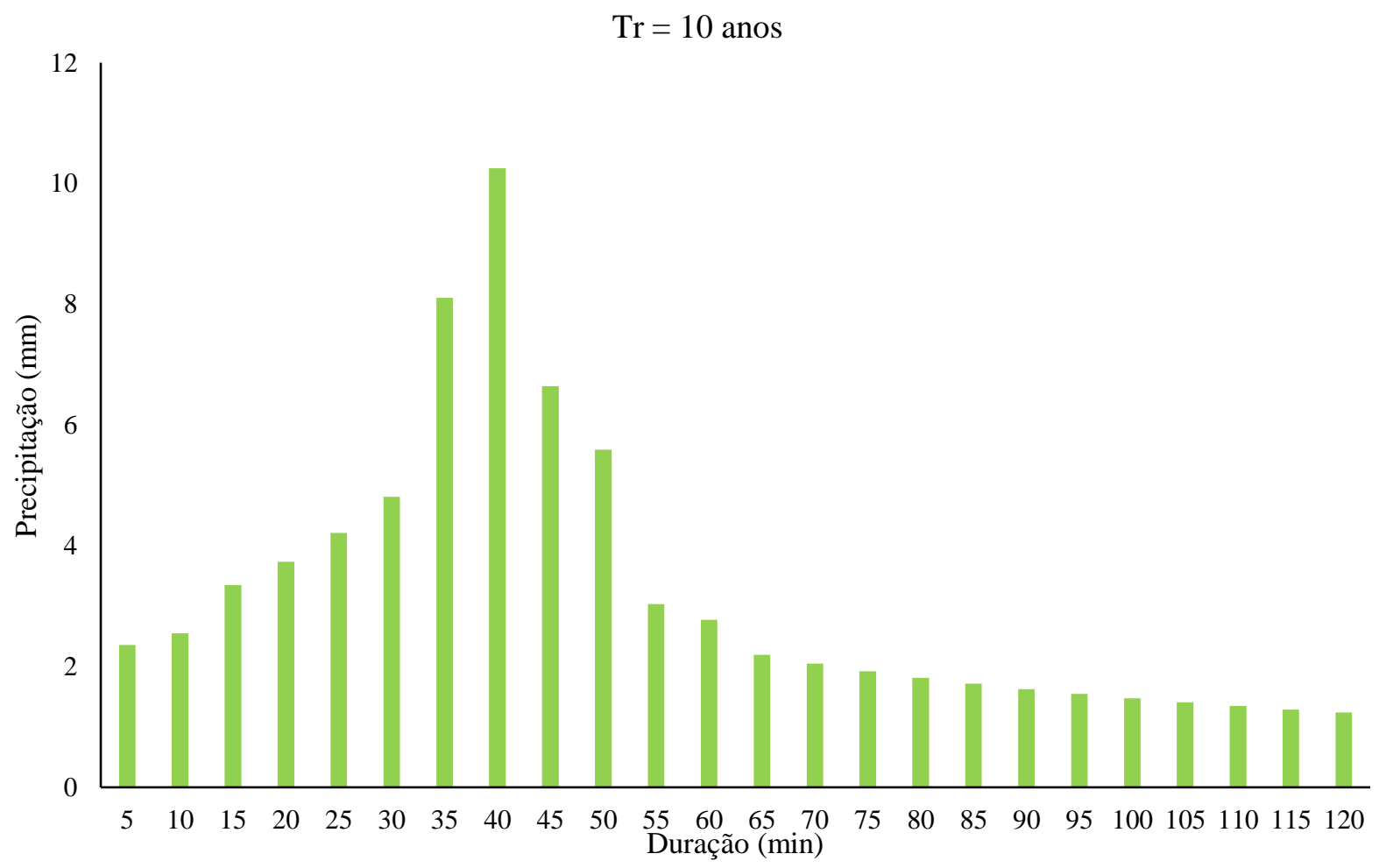

Fonte: Autores. 
Figura 10. Curvas de volume acumulado de precipitações para tempos de retorno de 5 anos e 10 anos e de infiltração de água no solo para toda a área de estudo.

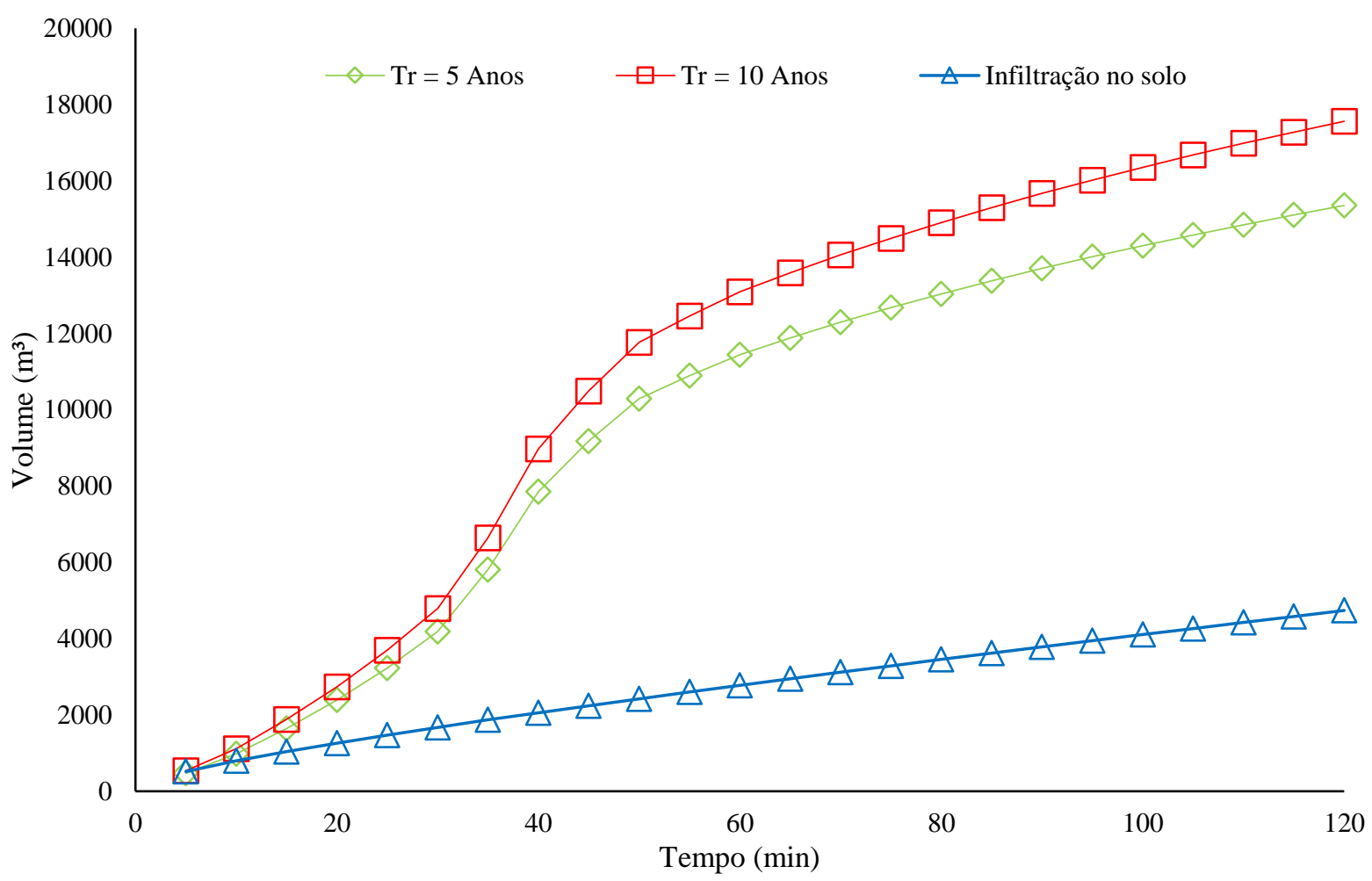

Fonte: Autores.

Na Figura 11 é possível visualizar os hidrogramas gerados pela precipitação (saldo positivo de água, no balanço hídrico) e pela infiltração (saldo negativo de água, no balanço hídrico). A área compreendida abaixo da curva de precipitação e acima da curva de infiltração compreende o escoamento superficial gerado na bacia. É perceptível que a infiltração tem participação mais ativa nos tempos iniciais do evento, perdendo significantemente seu efeito de amortecimento de escoamento superficial no decorrer do tempo, havendo contribuição tendendo a zero (comportamento de uma assíntota) nos períodos finais do hidrograma.

Figura 11. Hidrogramas de precipitação, para tempos de retorno de 10 anos e 5 anos, e infiltração para a bacia de estudo.

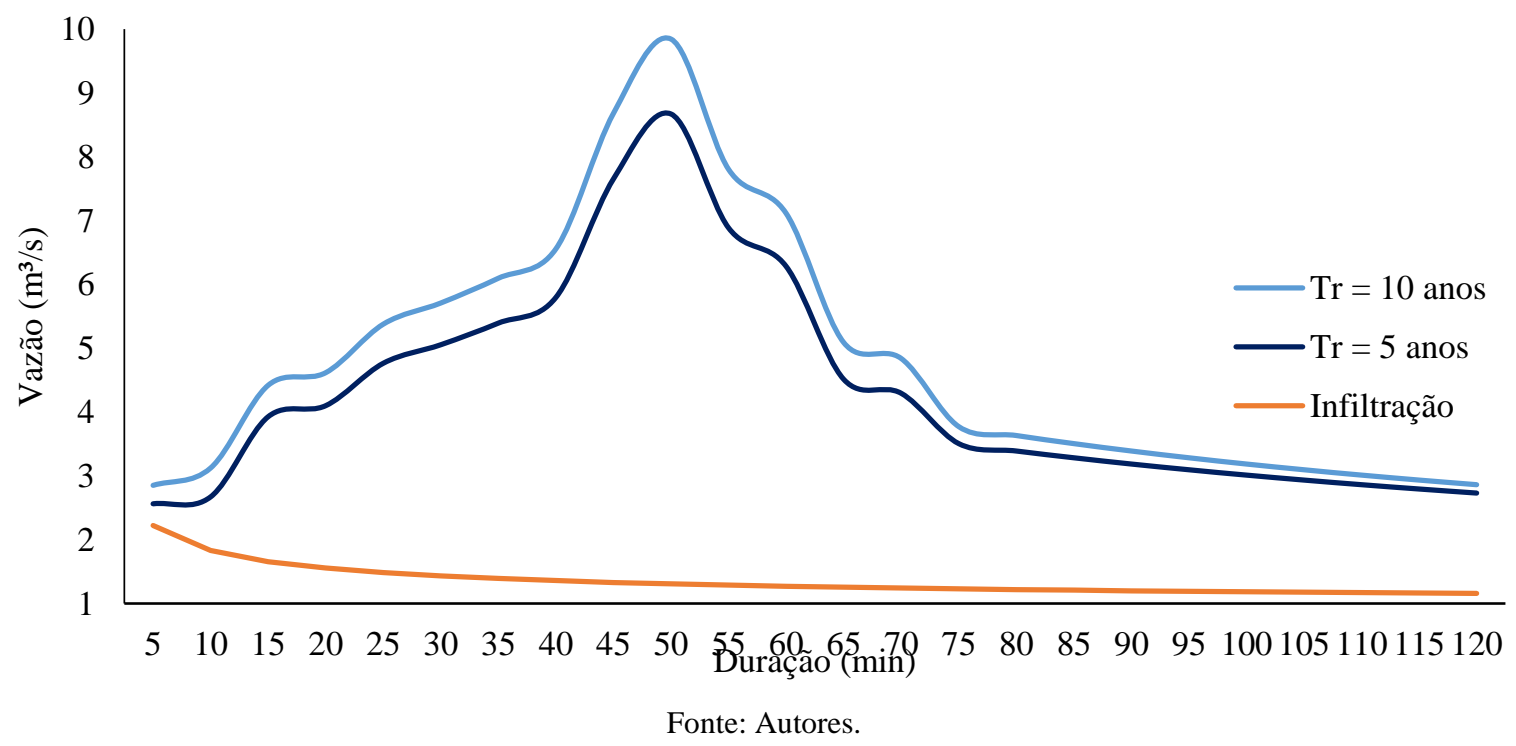




\section{Conclusão}

O método de ensaio de infiltração do anel simples, aliado ao modelo BEST de previsão de acumulo de água infiltrado no solo se mostra altamente eficiente para simulações práticas em solos arenosos, que depreendem baixo custo e tempo de utilização. O solo de parte do bairro da Madalena, no Recife, mostrou-se com alto potencial de retenção hídrica, podendo amortecer os efeitos de eventos pluviométricos consideráveis na área urbana em diversas ocasiões. Devido à baixa ocorrência deste, cerca de $17 \%$ da área total, sua atuação na drenagem urbana se mostrou bastante aquém do potencial, tendo seus efeitos praticamente desconsideráveis no hidrograma de projeto e mostrando baixa eficiência na retenção de volumes totais de água na bacia.

\section{Agradecimentos}

O primeiro autor agradece ao Programa de Pós-graduação em Tecnologias Energéticas e Nucleares (PROTEN) da UFPE, ao Conselho Nacional de Desenvolvimento Científico e Tecnológico (CNPq), pela disponibilização das bolsas. Todos os autores também agradecem ao Programa de Pós-graduação em Engenharia Civil (PEC) da Escola Politécnica de Pernambuco pelo apoio.

\section{Referências}

APAC - Agência Pernambucana de Águas e Clima. http://www.apac.pe.gov.br/meteorologia/monitoramento-pluvio.php.

Abreu, M. C., \& Tonello, K. C. (2017). Avaliação dos parâmetros hidrometeorológicos na bacia do Rio Sorocaba/SP. Revista Brasileira de Meteorologia, 32, http://dx.doi.org/10.1590/0102-778632120150164

Almeida, C. X., Centurion, J. F., Jorge, R. F., Barbosa, J. C., \& Andrioli, I. (2012). Funções de pedotransferência para a curva de resistência do solo à penetração. Revista Brasileira de Ciência do Solo, 36, 1745-1755. http://dx.doi.org/10.1590/S0100-06832008000600003

Barros, A. H. C., Lier, Q. J. V., Maia, A. H. N., \& Scarpare, F. V. (2013). Pedotransfer Functions to Estimate Water Rentention Parameters of Soils in Northeastern Brazil, Revista Brasileira de Ciências do Solo, 37, 379-391. http://dx.doi.org/10.1590/S0100-06832013000200009

Bidone, F., \& Tucci, C. E. M. (1995). Drenagem Urbana. Microdrenagem. Associação Brasileira de Recursos Hídricos, Porto Alegre.

Bielenki Júnior, C., Barbassa, A. P., Miranda, R. B., \& Mauad, F. F. (2016). Determinação de curva intensidade-duração-frequência por meio do emprego do método paramétrico de ajustamento de observações. Revista Brasileira de Climatologia, 19146-167. http://dx.doi.org/10.5380/abclima.v19i0.44083

Cecílio, R. A. (2005). Modelagem da infiltração da água no solo fundamentada na equação de Green-Ampt-Mein-Larson. Dissertação (Doutorado em Engenharia Agrícola) - Programa de pós-Graduação em Engenharia Agrícola, Universidade Federal de Viçosa, Viçosa.

Cemaden - Centro Nacional de Monitoramento e Alerta de Desastres Naturais. Disponível em: http://www.cemaden.gov.br/mapainterativo/\#. Acesso em: $29 / 12 / 2020$.

Costa, I. R. S., Teixeira, E. C., Costa, C. M., Barros, D. V., \& Silva, Q. D. (2016). Alagamentos e enxurradas: uma análise do uso e cobertura do solo na subbacia hidrográfica do riacho do turu São luís - MA. Revista Geonorte, Edição Especial 5, 7, 1-13.

EMBRAPA - Empresa Brasileira de Pesquisa Agropecuária (2018). Serviço de Produção de Informação - SPI. Sistema Brasileiro de Classificação de Solo. Brasília, 355p.

Teixeira, P. C., Donagemma, G. K., Fontana, A., Teixeira, W. G., \& EMBRAPA (2017), Manual de métodos de análise de solos.

Fagundes, L. (2002). Elaboração de índice ambiental urbano através da análise de densidade populacional e superfície impermeável em bacias hidrográficas. Dissertação (mestrado em planejamento urbano e regional) - departamento de urbanismo, Universidade Federal do Rio Grande do Sul, Porto Alegre.

Haverkamp, R., Ross, P. J., Smettem, K. R. J., \& Parlange, J. Y. (1994). Three-dimensional analysis of infiltration from the disc infiltrometer. 2. Phisically based infiltration equation. Water Resources Research, 30, 2931-2935. https://doi.org/10.1029/94WR01788

Holanda, M. A. C. R., Oliveira, D. B. C., Soares, W. A., \& Silva, S. R. (2020). Analysis of the Viability of Implementing Sustainable Proposals to Reduce Tunnel Flooding in Recife, Pernambuco. Revista Brasileira de Geografia Física, 13, 1904-1913. https://doi.org/10.26848/rbgf.v13.4.p1904-1913

Holanda, M. A. C. R., Soares, W. A., \& Oliveira, D. B. C. (2020). Predição do escoamento superficial e consequentes alagamentos em centros urbanos. Revista Ibero-Americana de Ciências Ambientais. 11, 1-11. https://doi.org/10.6008/CBPC2179-6858.2020.005.0001

INMET - Instituto Nacional de Meteorologia. http://www.inmet.gov.br/sim/gera_graficos.php.

Melo, T. A. T., Coutinho, A. P., Cabral, J. J. S. P., Cirilo, J. A., \& Antonino, A. C. D. (2013). Desafios para o manejo das águas pluviais na cidade do RecifeA contribuição da UFPE. Anais do XX Simpósio Brasileiro de Recursos Hídricos. Bento Gonçalves (RS). 
Lassabatère, L., Ângulo-Jaramillo, R., Ugalde, J.M.S., Cuenca, R., Braud, I., \& Haverkamp, R. (2006). Beerkan Estimation of Soil Transfer Parameters through Infiltration Experiments - BEST. Soil Science, v.70, p.521-532. https://doi.org/10.2136/sssaj2005.0026

Moura, E. F. S., \& Silva, S. R. (2015). Estudo do grau de impermeabilização do solo e propostas de técnicas de drenagem urbana sustentável em área do Recife - PE. Revista Nacional de Gerenciamento de Cidades, 3, 15. http://dx.doi.org/10.17271/231884723152015993

Netto, A. M., Lima, L. J. S., Antonino, A. C. D., Souza, E. S., \& Jaramillo, R. A. (2013). Caracterização hidrodinâmica e hidrodispersiva de um latossolo amarelo na microrregião do brejo paraibano. Revista Brasileira de Ciências do Solo, 37, http://dx.doi.org/10.1590/S0100-06832013000100009

Oliveira, D. B. C., \& Soares, W. A. (2017). Desempenho de modelos de infiltração de tridimensional de água no solo. Revista Diálogos, 2, 519-544. http://dx.doi.org/10.13115/2236-1499v2n18p519

Oliveira, D. B. C., Soares, W. A., \& Holanda, M. A. C. R. (2018). Análise de Desempenho de Modelos de Infiltração Unidimensional de Água no Solo. Revista Águas Subterrâneas. 3235-42. https://doi.org/10.14295/ras.v32i1.28947

Oliveira, T. H., Silva, J. E., Santos, M. O., Oliveira, J. S. S., \& Galvíncio, J. D. (2016). Evolução espaço-temporal da drenagem superficial e do processo de impermeabilização em Santo Amaro, Recife-PE. Revista Brasileira de Geografia Física, 8, .1571-1587. https://doi.org/10.5935/1984-2295.20150088

Porto, R. (1995). Drenagem Urbana. Escoamento Superficial Direto. Associação Brasileira de Recursos Hídricos.

Recife (1996), Lei n. 16.176, de 13 de abr. de 1996. Lei de uso e ocupação do solo da cidade do Recife. Recife, PE.

Silva, S. R., \& Araújo, G. R. S. (2013). Algoritmo para determinação da equação de chuvas intensas. Revista Brasileira de Geografia Física, 6, 1371-1383. https://doi.org/10.26848/rbgf.v6i5.233110

Silva Júnior, M. A. B., Silva, S. R., \& Soares, W. A. (2016). Parâmetros de infiltrabilidade em solo urbano para modelo chuva-vazão. Fórum Ambiental da Alta Paulista, 12, 135-143. http://dx.doi.org/10.17271/1980082712320161417

Smettem, K. R. J., Parlange, J. Y., Ross, P. J., \& Haverkamp, R. (1994). Three-dimensional analysis of infiltration from the disc infiltrometer. Part I. A capillarybased theory. Water Resource Research, 30, 2925-2929. https://doi.org/10.1029/94WR01787

Souza, C., Andrade, A. P., Lima, J. R. S., Antonino, A. C. D., Souza, E. S., \& Silva, E. F. (2013). Balanço hídrico da cultura da mamona sob condições de sequeiro. Revista Brasileira de Engenharia Agrícola e Ambiental, 123-10. http://dx.doi.org/10.1590/S1415-43662013000100001.

Soares, W. A., Almeida, C. A. B., \& Silva, H. M. (2005). Modelagem numérica do processo de infiltração de água para obtenção dos parâmetros hidrodinâmicos do solo. $5^{\circ}$ Encontro Regional de Matemática Aplicada e Computacional, Natal.

Souza, J. C. B. (2014). A influência do método de escolha do parâmetro " $C$ " na determinação de equações de chuvas intensas. Dissertação (Mestrado em Engenharia Civil) - Programa de pós-Graduação em Engenharia Civil, Universidade Federal de Pernambuco, Recife.

Souza, E. S., Antonino, A. C. D., Jaramillo, R. A., \& Netto, A. M. (2008). Caracterização hidrodinâmica de solos: aplicação do método Beerkan. Revista Brasileira de Engenharia Agrícola e Ambiental, 12, 2. http://dx.doi.org/10.1590/S1415-43662008000200004

Souza, B. R. F., Junior, C. A. S., Carvalho, L. A., Pellin, D. M. P., Correa, A. R. C., \& Sousa, R. A. C. (2014). Desempenho de amostradores de solo sob diferentes coberturas vegetais na região de Aquidauana-MS. Agrarian Academy, 1, 146-158.

Bertoni, J. C., \& Tucci, C. E. M. (2007). Precipitação.Hidrologia: ciência e aplicação. (4a ed.), Editora da UFRGS/ABRH. p. 177-241.

Van Genuchten, M.T. (1980). A closed form equation for predicting the hydraulic conductivity of unsaturated soils. Soil Science Society of America Journal, 44, 892-898. https://doi.org/10.2136/sssaj1980.03615995004400050002x 